\title{
Dynamics and behavior of a higher order rational recursive sequence
}

\author{
Elsayed M Elsayed ${ }^{1,2^{*}}$ and Mohammed M El-Dessoky ${ }^{1,2}$
}

\author{
* Correspondence: \\ emelsayed@mans.edu.eg \\ ${ }^{1}$ Mathematics Department, Faculty \\ of Science, King Abdulaziz \\ University, P. O. Box 80203, Jeddah \\ 21589, Saudi Arabia \\ Full list of author information is \\ available at the end of the article
}

\section{Abstract}

In this article we study the global convergence result, boundedness, and periodicity of solutions of the difference equation

$$
x_{n+1}=a x_{n-s}+\frac{b x_{n-l}+c x_{n-k}}{d x_{n-l}+e x_{n-k}} \quad n=0,1, \ldots,
$$

where the parameters $a, b, c, d$ and $e$ are positive real numbers and the initial conditions $x_{-t}, x_{-t+1}, \ldots, x_{-1}$ and $x_{0}$ are positive real numbers where $t=\max \{s, l, k\}$.

Mathematics Subject Classification: 39A10.

Keywords: stability, periodic solutions, boundedness, difference equations

\section{Introduction}

Difference equations appear as natural descriptions of observed evolution phenomena because most measurements of time evolving variables are discrete and as such these equations are in their own right important mathematical models. More importantly, difference equations also appear in the study of discretization methods for differential equations. Several results in the theory of difference equations have been obtained as more or less natural discrete analogues of corresponding results of differential equations.

The study of rational difference equations of order greater than one is quite challenging and rewarding because some prototypes for the development of the basic theory of the global behavior of nonlinear difference equations of order greater than one come from the results for rational difference equations. However, there have not been any effective general methods to deal with the global behavior of rational difference equations of order greater than one so far. Therefore, the study of rational difference equations of order greater than one is worth further consideration.

Recently there has been a lot of interest in studying the global attractivity, boundedness character, periodicity and the solution form of nonlinear difference equations. For some results in this area, for example: Agarwal and Elsayed [1] studied the global stability, periodicity character and gave the solution form of some special cases of the recursive sequence

$$
x_{n+1}=a x_{n}+\frac{b x_{n} x_{n-3}}{c x_{n-2}+d x_{n-3}} .
$$

(c) 2012 Elsayed and El-Dessoky; licensee Springer. This is an Open Access article distributed under the terms of the Creative Commons Attribution License (http://creativecommons.org/licenses/by/2.0), which permits unrestricted use, distribution, and reproduction in any medium, provided the original work is properly cited. 
Aloqeili [2] obtained the form of the solutions of the difference equation

$$
x_{n+1}=\frac{x_{n-1}}{a-x_{n} x_{n-1}} .
$$

Elabbasy et al. [3] investigated the global stability character, boundedness and the periodicity of solutions of the difference equation

$$
x_{n+1}=\frac{\alpha x_{n}+\beta x_{n-1}+\gamma x_{n-2}}{A x_{n}+B x_{n-1}+C x_{n-2}} .
$$

Elabbasy et al. [4] get the dynamics such that the global stability, periodicity character and gave the solution of special case of the following recursive sequence

$$
x_{n+1}=a x_{n}-\frac{b x_{n}}{c x_{n}-d x_{n-1}} .
$$

Elabbasy et al. [5] investigated the behavior of the difference equation especially global stability, boundedness, periodicity character and gave the solution of some special cases of the difference equation

$$
x_{n+1}=\frac{\alpha x_{n-k}}{\beta+\gamma \prod_{i=0}^{k} x_{n-i}} .
$$

El-Metwally et al. [6] dealed with the following difference equation

$$
y_{n+1}=\frac{y_{n-(2 k+1)}+p}{y_{n-(2 k+1)}+q y_{n-2 l}} .
$$

Saleh and Aloqeili [7] investigated the difference equation

$$
y_{n+1}=A+\frac{y_{n}}{y_{n-k}} .
$$

Simsek et al. [8] obtained the solution of the difference equation

$$
x_{n+1}=\frac{x_{n-3}}{1+x_{n-1}} .
$$

Yalçınkaya $[9,10]$ considered the dynamics of the difference equations

$$
x_{n+1}=\frac{a x_{n-k}}{b+c x_{n}^{p}}, \quad x_{n+1}=\alpha+\frac{x_{n-m}}{x_{n}^{k}} .
$$

Zayed and El-Moneam [11,12] studied the behavior of the following rational recursive sequences

$$
x_{n+1}=a x_{n}-\frac{b x_{n}}{c x_{n}-d x_{n-k}}, \quad x_{n+1}=\frac{\alpha+\beta x_{n}+\gamma x_{n-1}}{A+B x_{n}+C x_{n-1}} .
$$

Other related results on rational difference equations can be found in [1-45].

Our goal in this article is to investigate the global stability character and the periodicity of solutions of the recursive sequence

$$
x_{n+1}=a x_{n-s}+\frac{b x_{n-l}+c x_{n-k}}{d x_{n-l}+e x_{n-k}}
$$


where the parameters $a, b, c, d$ and $e$ are positive real numbers and the initial conditions $x_{-t}, x_{-t+1}, \ldots, x_{-1}$ and $x_{0}$ are positive real numbers where $t=\max \{s, l, k\}$.

\section{Some basic properties and definitions}

Here, we recall some basic definitions and some theorems that we need in the sequel.

Let $I$ be some interval of real numbers and let

$$
F: I^{k+1} \rightarrow I,
$$

be a continuously differentiable function. Then for every set of initial conditions $x_{-k}$, $x_{-k+1}, \ldots, x_{0} \in I$, the difference equation

$$
x_{n+1}=F\left(x_{n}, x_{n-1}, \ldots, x_{n-k}\right), \quad n=0,1, \ldots,
$$

has a unique solution $\left\{x_{n}\right\}_{n=-k}^{\infty}$.

Definition 1 (Equilibrium point)

A point $\bar{x} \in I$ is called an equilibrium point of Equation (2) if

$$
\bar{x}=F(\bar{x}, \bar{x}, \ldots, \bar{x}) .
$$

That is, $x_{n}=\bar{x}$ for $n \geq 0$, is a solution of Equation (2), or equivalently, $\bar{x}$ is a fixed point of $F$.

\section{Definition 2 (Periodicity)}

A sequence $\left\{x_{n}\right\}_{n=-k}^{\infty}$ is said to be periodic with period $p$ if $x_{n+p}=x_{n}$ for all $n \geq-k$.

Definition 3 (Stability)

(i) The equilibrium point $\bar{x}$ of Equation (2) is locally stable if for every $\varepsilon>0$, there exists $\delta>0$ such that for all $x_{-k}, x_{-k+1}, \ldots, x_{-1}, x_{0} \in I$ with

$$
\left|x_{-k}-\bar{x}\right|+\left|x_{-k+1}-\bar{x}\right|+\cdots+\left|x_{0}-\bar{x}\right|<\delta,
$$

we have

$$
\left|x_{n}-\bar{x}\right|<\epsilon \text { for all } n \geq-k .
$$

(ii) The equilibrium point $\bar{x}$ of Equation (2) is locally asymptotically stable if $\bar{x}$ is locally stable solution of Equation (2) and there exists $\gamma>0$, such that for all $x_{-k}, x_{-k+1}$, . $\ldots, x_{-1}, x_{0} \in I$ with

$$
\left|x_{-k}-\bar{x}\right|+\left|x_{-k+1}-\bar{x}\right|+\cdots+\left|x_{0}-\bar{x}\right|<\gamma,
$$

we have

$$
\lim _{n \rightarrow \infty} x_{n}=\bar{x} .
$$

(iii) The equilibrium point $\bar{x}$ of Equation (2) is global attractor if for all $x_{-k}, x_{-k+1}, \ldots$ , $x_{-1}, x_{0} \in I$, we have

$$
\lim _{n \rightarrow \infty} x_{n}=\bar{x}
$$

(iv) The equilibrium point $\bar{x}$ of Equation (2) is globally asymptotically stable if $\bar{x}$ is locally stable, and $\bar{x}$ is also a global attractor of Equation (2).

(v) The equilibrium point $\bar{x}$ of Equation (2) is unstable if $\bar{x}$ is not locally stable. 
The linearized equation of Equation (2) about the equilibrium $\bar{x}$ is the linear difference equation

$$
y_{n+1}=\sum_{i=0}^{k} \frac{\partial F(\bar{x}, \bar{x}, \ldots, \bar{x})}{\partial x_{n-i}} y_{n-i} .
$$

Theorem A: [34] Assume that $p_{i} \in R, i=1,2, \ldots$ and $k \in\{0,1,2, \ldots\}$. Then

$$
\sum_{i=1}^{k}\left|p_{i}\right|<1
$$

is a sufficient condition for the asymptotic stability of the difference equation

$$
y_{n+k}+p_{1} y_{n+k-1}+\cdots+p_{k} y_{n}=0, \quad n=0,1, \ldots
$$

Consider the following equation

$$
x_{n+1}=g\left(x_{n}, x_{n-1}, x_{n-2}\right) \text {. }
$$

The following two theorems will be useful for the proof of our results in this article.

Theorem B: [35] Let $[\alpha, \beta]$ be an interval of real numbers and assume that

$$
g:[\alpha, \beta]^{3} \rightarrow[\alpha, \beta]
$$

is a continuous function satisfying the following properties:

(a) $g(x, y, z)$ is non-decreasing in $x$ and $y$ in $[\alpha, \beta]$ for each $z \in[\alpha, \beta]$, and is nonincreasing in $z \in[\alpha, \beta]$ for each $x$ and $y$ in $[\alpha, \beta]$;

(b) If $(m, M) \in[\alpha, \beta] \times[\alpha, \beta]$ is a solution of the system

$$
M=g(M, M, m) \quad \text { and } \quad m=g(m, m, M),
$$

then

$$
m=M .
$$

Then Equation (5) has a unique equilibrium $\bar{x} \in[\alpha, \beta]$ and every solution of Equation (5) converges to $\bar{x}$.

Theorem C: [35] Let $[\alpha, \beta]$ be an interval of real numbers and assume that

$$
g:[\alpha, \beta]^{3} \rightarrow[\alpha, \beta]
$$

is a continuous function satisfying the following properties:

(a) $g(x, y, z)$ is non-decreasing in $x$ and $z$ in $[\alpha, \beta]$ for each $y \in[\alpha, \beta]$, and is nonincreasing in $y \in[\alpha, \beta]$ for each $x$ and $z$ in $[\alpha, \beta]$;

(b) If $(m, M) \in[\alpha, \beta] \times[\alpha, \beta]$ is a solution of the system

$$
M=g(M, m, M) \text { and } m=g(m, M, m),
$$

then

$$
m=M .
$$

Then Equation (5) has a unique equilibrium $\bar{x} \in[\alpha, \beta]$ and every solution of Equation (5) converges to $\bar{x}$. 
The article proceeds as follows. In Section 3 we show that the equilibrium point of Equation (1) is locally asymptotically stable when $2|(b e-d c)|<(d+e)(b+c)$. In Section 4 we prove that the solution is bounded when $a<1$ and the solution of Equation (1) is unbounded if $a>1$. In Section 5 we prove that the there exists a period two solution of Equation (1). In Section 6 we prove that the equilibrium point of Equation (1) is global attractor. Finally, we give numerical examples of some special cases of Equation (1) and draw it by using Matlab.

\section{Local Stability of the equilibrium point of Equation (1)}

This section deals with study the local stability character of the equilibrium point of Equation (1).

Theorem 1 Assume that

$$
2|(b e-d c)|<(d+e)(b+c) .
$$

Then the positive equilibrium point of Equation (1) is locally asymptotically stable.

Proof. Equation (1) has equilibrium point and is given by

$$
\bar{x}=a \bar{x}+\frac{b+c}{d+e} .
$$

If $a<1$, then the only positive equilibrium point of Equation (1) is given by

$$
\bar{x}=\frac{b+c}{(1-a)(d+e)} .
$$

Let $f:(0, \infty)^{3} \rightarrow(0, \infty)$ be a continuous function defined by

$$
f(u, v, w)=a u+\frac{b v+c w}{d v+e w} .
$$

Therefore it follows that

$$
\begin{aligned}
& \frac{\partial f(u, v, w)}{\partial u}=a \\
& \frac{\partial f(u, v, w)}{\partial v}=\frac{(b e-d c) w}{(d v+e w)^{2}}, \\
& \frac{\partial f(u, v, w)}{\partial w}=\frac{(d c-b e) u}{(d v+e w)^{2}} .
\end{aligned}
$$

Then we see that

$$
\begin{aligned}
& \frac{\partial f(\bar{x}, \bar{x}, \bar{x})}{\partial u}=a=-a_{2}, \\
& \frac{\partial f(\bar{x}, \bar{x}, \bar{x})}{\partial v}=\frac{(b e-d c)}{(d+e)^{2} \bar{x}}=\frac{(b e-d c)(1-a)}{(d+e)(b+c)}=-a_{1}, \\
& \frac{\partial f(\bar{x}, \bar{x}, \bar{x})}{\partial w}=\frac{(d c-b e)}{(d+e)^{2} \bar{x}}=\frac{(d c-b e)(1-a)}{(d+e)(b+c)}=-a_{0} .
\end{aligned}
$$

Then the linearized equation of Equation (1) about $\bar{x}$ is

$$
y_{n+1}+a_{2} y_{n-s}+a_{1} y_{n-l}+a_{0} y_{n-k}=0,
$$

whose characteristic equation is

$$
\lambda^{k+1}+a_{2} \lambda^{k-s}+a_{1} \lambda^{k-l}+a_{0}=0 .
$$


It follows by Theorem A that, Equation (7) is asymptotically stable if all roots of Equation (8) lie in the open disc $|\lambda|<1$ that is if

$$
\begin{gathered}
\left|a_{2}\right|+\left|a_{1}\right|+\left|a_{0}\right|<1 . \\
|a|+\left|\frac{(b e-d c)(1-a)}{(d+e)(b+c)}\right|+\left|\frac{(d c-b e)(1-a)}{(d+e)(b+c)}\right|<1,
\end{gathered}
$$

and so

$$
2\left|\frac{(b e-d c)(1-a)}{(d+e)(b+c)}\right|<(1-a), \quad a<1,
$$

or

$$
2|b e-d c|<(d+e)(b+c) .
$$

The proof is complete.

\section{Existence of bounded and unbounded solutions of Equations (1)}

Here we study the boundedness nature of solutions of Equation (1).

Theorem 2 Every solution of Equation (1) is bounded if a $<1$.

Proof. Let $\left\{x_{n}\right\}_{n=-t}^{\infty}$ be a solution of Equation (1). It follows from Equation (1) that

$$
x_{n+1}=a x_{n-s}+\frac{b x_{n-l}+c x_{n-k}}{d x_{n-l}+e x_{n-k}}=a x_{n-s}+\frac{b x_{n-l}}{d x_{n-l}+e x_{n-k}}+\frac{c x_{n-k}}{d x_{n-l}+e x_{n-k}} .
$$

Then

$$
x_{n+1} \leq a x_{n-s}+\frac{b x_{n-l}}{d x_{n-l}}+\frac{c x_{n-k}}{e x_{n-k}}=a x_{n-s}+\frac{b}{d}+\frac{c}{e} \text { for all } n \geq 1 .
$$

By using a comparison, we can write the right hand side as follows

$$
y_{n+1}=a y_{n-s}+\frac{b}{d}+\frac{c}{e}
$$

then

$$
\gamma_{s n+i}=a^{n} \gamma_{s+i}+\text { constant }, i=0,1, \ldots, s,
$$

and this equation is locally asymptotically stable because $a<1$, and converges to the equilibrium point $\bar{y}=\frac{b e+c d}{d e(1-a)}$.

Therefore

$$
\limsup _{n \rightarrow \infty} x_{n} \leq \frac{b e+c d}{d e(1-a)} .
$$

Thus the solution is bounded.

Theorem 3 Every solution of Equation (1) is unbounded if $a>1$.

Proof. Let $\left\{x_{n}\right\}_{n=-t}^{\infty}$ be a solution of Equation (1). Then from Equation (1) we see that

$$
x_{n+1}=a x_{n-s}+\frac{b x_{n-l}+c x_{n-k}}{d x_{n-l}+e x_{n-k}}>a x_{n-s} \text { for all } n \geq 1 .
$$


We see that the right hand side can write as follows

$$
y_{n+1}=a y_{n-s} \Rightarrow y_{s n+i}=a^{n} \gamma_{s+i}, \quad i=0,1, \ldots, s,
$$

and this equation is unstable because $a>1$, and $\lim _{n \rightarrow \infty} y_{n}=\infty$. Then by using ratio test $\left\{x_{n}\right\}_{n=-t}^{\infty}$ is unbounded from above.

\section{Existence of periodic solutions}

In this section we study the existence of periodic solutions of Equation (1). The following theorem states the necessary and sufficient conditions that this equation has periodic solutions of prime period two.

Theorem 4 Equation (1) has positive prime period two solutions if and only if

$(i)(b-c)(d-e)(1+a)+4(b a e+c d)>0, d>e, b>c$ and $-o d d, k, s-e v e n$.

(ii) $(c-b)(e-d)(1+a)+4(a c d+b e)>0, e>d, c>b$ and $k-o d d, l, s-e v e n$.

(iii) $(c-b)(d-e)-4 b e>0, d>e, c>b, a<1$, and $k, s-o d d, l-e v e n$.

(iv) $(b-c)(e-d)-4 c d>0, e>d, b>c, a<1$, and $l, s-o d d, k-e v e n$.

Proof. We prove that when $l$-odd, $k, s$ - even (the other cases are similar and will be omitted.)

First suppose that there exists a prime period two solution

$$
\ldots, p, q, p, q, \ldots,
$$

of Equation (1). We will prove that Condition (i) holds.

We see from Equation (1) when $l$ - odd, $k$ - even that

$$
p=a q+\frac{b p+c q}{d p+e q}
$$

and

$$
q=a p+\frac{b q+c p}{d q+e p} .
$$

Then

$$
d p^{2}+e p q=a d p q+a e q^{2}+b p+c q,
$$

and

$$
d q^{2}+e p q=a d p q+a e p^{2}+b q+c p .
$$

Subtracting (9) from (10) gives

$$
d\left(p^{2}-q^{2}\right)=-a e\left(p^{2}-q^{2}\right)+(b-c)(p-q) .
$$

Since $p \neq q$, it follows that

$$
p+q=\frac{(b-c)}{(d+a e)} .
$$


Again, adding (9) and (10) yields

$$
\begin{gathered}
d\left(p^{2}+q^{2}\right)+2 e p q=2 a d p q+a e\left(p^{2}+q^{2}\right)+(b+c)(p+q) \\
(d-a e)\left(p^{2}+q^{2}\right)+2(e-a d) p q=(b+c)(p+q) .
\end{gathered}
$$

It follows by (11), (12) and the relation

$$
p^{2}+q^{2}=(p+q)^{2}-2 p q \text { for all } p, q \in R,
$$

that

$$
2(e-d)(1+a) p q=\frac{2(b a e+c d)(b-c)}{(d+a e)^{2}} .
$$

Thus

$$
p q=\frac{(b a e+c d)(b-c)}{(d+a e)^{2}(e-d)(1+a)} .
$$

Now it is clear from Equations (11) and (13) that $p$ and $q$ are the two distinct roots of the quadratic equation

$$
\begin{aligned}
& t^{2}-\left(\frac{(b-c)}{(d+a e)}\right) t+\left(\frac{(b a e+c d)(b-c)}{(d+a e)^{2}(e-d)(1+a)}\right)=0 \\
& (d+a e) t^{2}-(b-c) t+\left(\frac{(b a e+c d)(b-c)}{(d+a e)(e-d)(1+a)}\right)=0
\end{aligned}
$$

and so

$$
[b-c]^{2}-\frac{4(b a e+c d)(b-c)}{(e-d)(1+a)}>0,
$$

or

$$
\begin{gathered}
{[b-c]^{2}+\frac{4(b a e+c d)(b-c)}{(d-e)(1+a)}>0 .} \\
(b-c)(d-e)(1+a)+4(b a e+c d)>0 .
\end{gathered}
$$

Therefore inequalities (i) holds.

Second suppose that inequalities (i) is true. We will show that Equation (1) has a prime period two solution.

Assume that

$$
p=\frac{b-c+\zeta}{2(d+a e)}
$$

and

$$
q=\frac{b-c-\zeta}{2(d+a e)}
$$

where $\zeta=\sqrt{[b-c]^{2}-\frac{4(b a e+c d)(b-c)}{(e-d)(1+a)}}$. 
We see from inequalities (i) that

$$
(b-c)(d-e)(1+a)+4(b a e+c d)>0, b>c, d>e,
$$

which equivalents to

$$
(b-c)^{2}>\frac{4(b a e+c d)(b-c)}{(e-d)(1+a)} .
$$

Therefore $p$ and $q$ are distinct real numbers.

Set

$$
x_{-s}=q, x_{-l}=p, x_{-k}=q, \ldots, x_{-2}=q, x_{-1}=p \text { and } x_{0}=q .
$$

We wish to show that

$$
x_{1}=x_{-1}=p \text { and } x_{2}=x_{0}=q .
$$

It follows from Equation (1) that

$$
x_{1}=a q+\frac{b p+c q}{d p+e q}=a\left(\frac{b-c-\zeta}{2(d+a e)}\right)+\frac{b\left(\frac{b-c+\zeta}{2(d+a e)}\right)+c\left(\frac{b-c-\zeta}{2(d+a e)}\right)}{d\left(\frac{b-c+\zeta}{2(d+a e)}\right)+e\left(\frac{b-c-\zeta}{2(d+a e)}\right)} .
$$

Dividing the denominator and numerator by $2(d+a e)$ gives

$$
\begin{aligned}
x_{1} & =\frac{a b-a c-a \zeta}{2(d+a e)}+\frac{b(b-c+\zeta)+c(b-c-\zeta)}{d(b-c+\zeta)+e(b-c-\zeta)} \\
& =\frac{a b-a c-a \zeta}{2(d+a e)}+\frac{(b-c)[(b+c)+\zeta]}{(d+e)(b-c)+(d-e) \zeta} .
\end{aligned}
$$

Multiplying the denominator and numerator of the right side by $(d+e)(b-c)-(d-$ e) $\zeta$ gives

$$
\begin{aligned}
x_{1}= & \frac{a b-a c-a \zeta}{2(d+a e)}+\frac{(b-c)[(b+c)+\zeta][(d+e)(b-c)-(d-e) \zeta]}{[(d+e)(b-c)+(d-e) \zeta][(d+e)(b-c)-(d-e) \zeta]} \\
= & \frac{a b-a c-a \zeta}{2(d+a e)} \\
& +\frac{(b-c)\left\{(d+e)\left(b^{2}-c^{2}\right)+\zeta[(d+e)(b-c)-(d-e)(b+c)]-(d-e) \zeta^{2}\right\}}{(d+e)^{2}(b-c)^{2}-(d-e)^{2} \zeta^{2}} \\
= & \frac{a b-a c-a \zeta}{2(d+a e)} \\
& +\frac{(b-c)\left\{(d+e)\left(b^{2}-c^{2}\right)+2 \zeta(e b-c d)-(d-e)\left([b-c]^{2}-\frac{4(b a e+c d)(b-c)}{(e-d)(1+a)}\right)\right\}}{(d+e)^{2}(b-c)^{2}-(d-e)^{2}\left([b-c]^{2}-\frac{4(b a e+c d)(b-c)}{(e-d)(1+a)}\right)} \\
= & \frac{a b-a c-a \zeta}{2(d+a e)} \\
& +\frac{(b-c)\left\{(d+e)\left(b^{2}-c^{2}\right)+2 \zeta(e b-c d)-(d-e)(b-c)^{2}-\frac{4(b a e+c d)(b-c)}{(1+a)}\right\}}{(d+e)^{2}(b-c)^{2}-(d-e)^{2}\left([b-c]^{2}-\frac{4(b a e+c d)(b-c)}{(e-d)(1+a)}\right)} \\
= & \frac{a b-a c-a \zeta}{2(d+a e)}+\frac{(b-c)\left\{2(b-c)\left[d c+e b-\frac{2(b a e+c d)}{(1+a)}\right]+2 \zeta(e b-c d)\right\}}{4(b-c)\left[e d(b-c)+\frac{(e-d)(b a e+c d)}{(1+a)}\right]} .
\end{aligned}
$$


Multiplying the denominator and numerator of the right side by $(1+a)$ we obtain

$$
\begin{gathered}
x_{1}=\frac{a b-a c-a \zeta}{2(d+a e)}+\frac{(b-c)[(d c+e b)(1+a)-2(b a e+c d)]+\zeta(1+a)(e b-c d)}{2[e d(b-c)(1+a)+(e-d)(b a e+c d)]} \\
=\frac{a b-a c-a \zeta}{2(d+a e)}+\frac{(b-c)(e b-d c)(1-a)+\zeta(1+a)(e b-c d)}{2[e d(b-c)(1+a)+(e-d)(b a e+c d)]} \\
=\frac{a b-a c-a \zeta}{2(d+a e)}+\frac{(e b-d c)\{(b-c)(1-a)+\zeta(1+a)\}}{2(e b-c d)(d+a e)} \\
=\frac{a b-a c-a \zeta}{2(d+a e)}+\frac{(b-c)(1-a)+\zeta(1+a)}{2(d+a e)} \\
=\frac{a b-a c-a \zeta+(b-c)(1-a)+\zeta(1+a)}{2(d+a e)}=\frac{b-c+\zeta}{2(d+a e)}=p .
\end{gathered}
$$

Similarly as before one can easily show that

$$
x_{2}=q \text {. }
$$

Then it follows by induction that

$$
x_{2 n}=q \text { and } x_{2 n+1}=p \text { for all } n \geq-1 .
$$

Thus Equation (1) has the prime period two solution

$$
\ldots, p, q, p, q, \ldots,
$$

where $p$ and $q$ are the distinct roots of the quadratic equation (14) and the proof is complete.

Lemma 5 If $l, k, s$ - even. Then there exists a prime period two solutions if and only if $a=-1$.

Proof. First suppose that there exists a prime period two solution

$$
\ldots, p, q, p, q, \ldots,
$$

then we see from Equation (1) that when $l, k, s$ - even

$$
p=a q+\frac{b+c}{d+e}
$$

and

$$
q=a p+\frac{b+c}{d+e} .
$$

Subtracting (15) from (16) gives

$$
p-q=-a(p-q) \text {. }
$$

Since $p \neq q$, it follows that

$$
a=-1 \text {. }
$$

Again, adding (15) and (16) yields

$$
p+q=\frac{b+c}{d+e} .
$$


If we take

$$
p=\frac{b}{d+e}, \quad q=\frac{c}{d+e}, \quad \text { if } \quad b \neq c .
$$

Set

$$
x_{-s}=q, x_{-l}=p, x_{-k}=q, \ldots, x_{-2}=q, x_{-1}=p \text { and } x_{0}=q .
$$

We wish to show that

$$
x_{1}=x_{-1}=p \text { and } x_{2}=x_{0}=q .
$$

It follows from Equation (1) that

$$
x_{1}=a q+\frac{b q+c q}{d q+e q}=a\left(\frac{c}{d+e}\right)+\frac{b+c}{d+e}=\frac{-c}{d+e}+\frac{b+c}{d+e}=\frac{b}{d+e}=p .
$$

Similarly as before one can easily show that

$$
x_{2}=q .
$$

Then it follows by induction that

$$
x_{2 n}=q \text { and } x_{2 n+1}=p \text { for all } n \geq-1 .
$$

Thus Equation (1) has the prime period two solution

$$
\ldots, p, q, p, q, \ldots,
$$

and the proof is complete.

Lemma 6 If $l, k$-odd, s-even. Then there exists a positive prime period two solutions if and only if $a=-1$.

Proof. The proof as the previous Lemma and it will be omitted.

\section{Global attractivity of the equilibrium point of Equation (1)}

In this section we investigate the global asymptotic stability of Equation (1).

Lemma 7 For any values of the quotient $\frac{b}{d}$ and $\frac{c}{e}$, the function $f(u, v, w)$ defined by

Equation (6) has the monotonicity behavior in its two arguments.

Proof. The proof follows by some computations and it will be omitted.

Theorem 8 The equilibrium point $\bar{x}$ is a global attractor of Equation (1) if one of the following statements holds

(1) $b e \geq d c$ and $c \geq b$.

(2) $b e \leq d c$ and $c \leq b$.

Proof. Let $\alpha$ and $\beta$ be a real numbers and assume that $g:[\alpha, \beta]^{3} \rightarrow[\alpha, \beta]$ be a function defined by

$$
g(u, v, w)=a u+\frac{b v+c w}{d v+e w} .
$$


Then

$$
\begin{aligned}
& \frac{\partial g(u, v, w)}{\partial u}=a \\
& \frac{\partial g(u, v, w)}{\partial v}=\frac{(b e-d c) w}{(d v+e w)^{2}} \\
& \frac{\partial g(u, v, w)}{\partial w}=\frac{(d c-b e) u}{(d v+e w)^{2}}
\end{aligned}
$$

We consider the two cases:-

Case (1) Assume that (17) is true, then we can easily see that the function $g(u, v, w)$ increasing in $u, v$ and decreasing in $w$.

Suppose that $(m, M)$ is a solution of the system $M=g(M, M, m)$ and $m=g(m, m$, $M)$. Then from Equation (1), we see that

$$
M=a M+\frac{b M+c m}{d M+e m}, \quad m=a m+\frac{b m+c M}{d m+e M},
$$

or

$$
M(1-a)=\frac{b M+c m}{d M+e m}, \quad m(1-a)=\frac{b m+c M}{d m+e M},
$$

then

$$
d(1-a) M^{2}+e(1-a) M m=b M+c m, \quad d(1-a) m^{2}+e(1-a) M m=b m+c M .
$$

Subtracting this two equations we obtain

$$
(M-m)\{d(1-a)(M+m)+(c-b)\}=0,
$$

under the conditions $c \geq b, a<1$, we see that

$$
M=m .
$$

It follows by Theorem B that $\bar{x}$ is a global attractor of Equation (1) and then the proof is complete.

Case (2) Assume that (18) is true, let $\alpha$ and $\beta$ be a real numbers and assume that $g$ : $[\alpha, \beta]^{3} \rightarrow[\alpha, \beta]$ be a function defined by $g(u, v, w)=a u+\frac{b v+c w}{d v+e w}$, then we can easily see that the function $g(u, v, w)$ increasing in $u, w$ and decreasing in $v$.

Suppose that $(m, M)$ is a solution of the system $M=g(M, m, M)$ and $m=g(m, M$, $m)$. Then from Equation (1), we see that

$$
M=a M+\frac{b m+c M}{d m+e M}, \quad m=a m+\frac{b M+c m}{d M+e m},
$$

or

$$
M(1-a)=\frac{b m+c M}{d m+e M}, \quad m(1-a)=\frac{b M+c m}{d M+e m}
$$

then

$$
d(1-a) M m+e(1-a) M^{2}=b m+c M, \quad d(1-a) m M+e(1-a) m^{2}=b M+c m .
$$


Subtracting we obtain

$$
(M-m)\{e(1-a)(M+m)+(b-c)\}=0,
$$

under the conditions $b \geq c, a<1$, we see that

$$
M=m \text {. }
$$

It follows by Theorem $C$ that $\bar{x}$ is a global attractor of Equation (1) and then the proof is complete.

\section{Numerical examples}

For confirming the results of this article, we consider numerical examples which represent different types of solutions to Equation (1).

Example 1. We assume $l=3, k=4, s=0, x_{-4}=4, x_{-3}=13, x_{-2}=9, x_{-1}=15, x_{0}=2$, $a=0.9, b=5, c=2, d=3, e=1$. See Figure 1 .

Example 2. See Figure 2, since $l=3, k=4, s=2, x_{-4}=3, x_{-3}=11, x_{-2}=9, x_{-1}=5$, $x_{0}=7, a=0.8, b=5, c=4, d=3, e=2$.

Example 3. We consider $l=2, k=3, s=4, x_{-3}=7, x_{-3}=1, x_{-2}=4, x_{-1}=11, x_{0}=3$, $a=1.5, b=2, c=3, d=3, e=2$. See Figure 3 .

Example 4. See Figure 4, since $l=1, k=3, s=2, x_{-3}=b /(d+e), x_{-2}=c /(d+e), x_{-1}=$ $b /(d+e), x_{0}=c /(d+e), a=-1, b=5, c=6, d=3, e=4$.

Example 5. Figure 5 shows the solutions when $l=1, k=2, s=0, a=0.8, b=0.5, c$ $=0.2, d=5, e=0.6, x_{-2}=q, x_{-1}=p, x_{0}=q$.

$$
\left(\text { Since } p, q=\frac{b-c \pm \sqrt{[b-c]^{2}-\frac{4(b a e+c d)(b-c)}{(e-d)(1+a)}}}{2(d+a e)}\right) \text {. }
$$

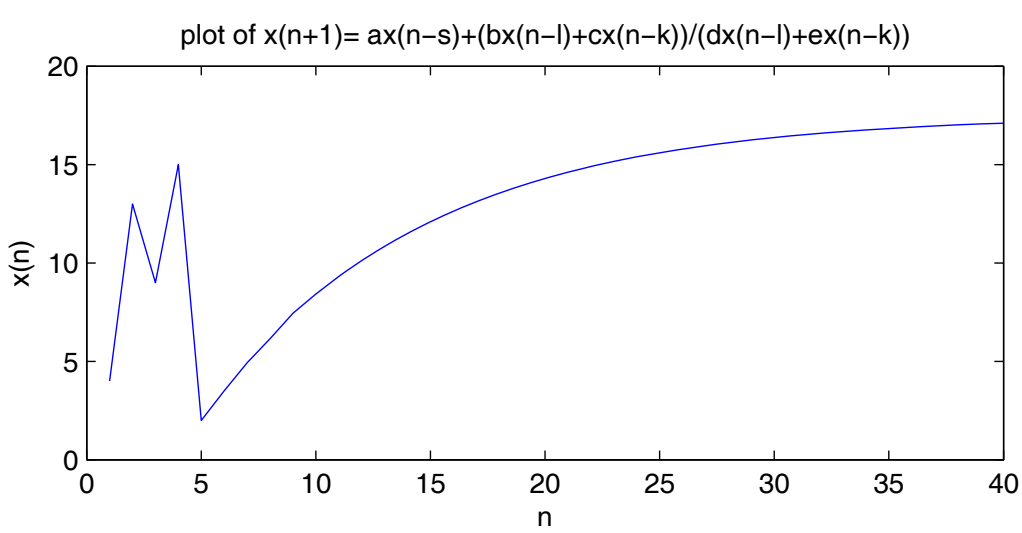

Figure 1 This figure shows the solution of Equation (1) with $I=3, k=4, s=0, x_{-4}=4, x_{-3}=13, x_{-2}$ $=9, x_{-1}=15, x_{0}=2, a=0.9, b=5, c=2, d=3, e=1$. 


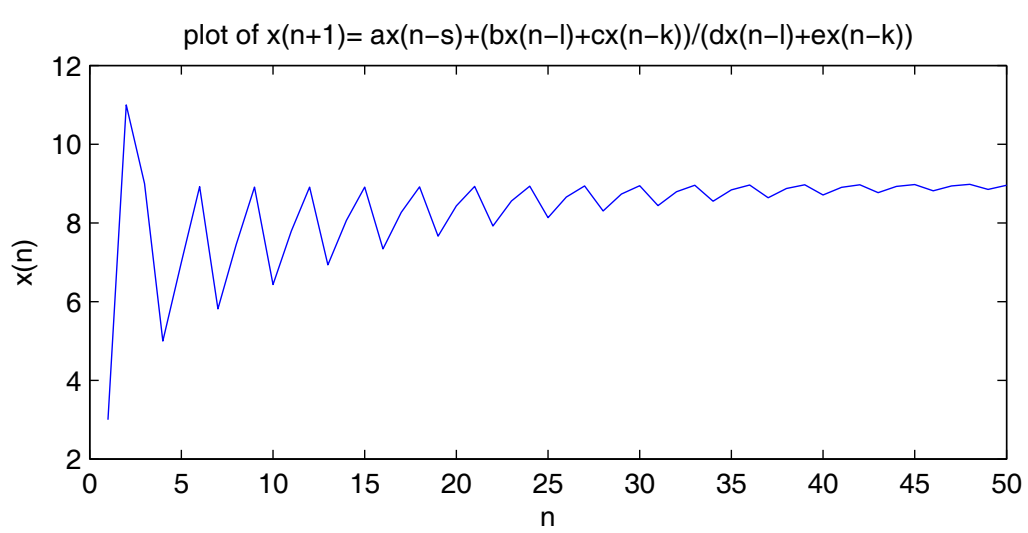

Figure 2 This figure shows the solution of $x_{n+1}=0.8 x_{n-2}+\frac{5 x_{n-3}+4 x_{n-4}}{3 x_{n-3}+2 x_{n-4}}$, with the initial conditions $x_{-4}=3, x_{-3}=11, x_{-2}=9, x_{-1}=5, x_{0}=7$.

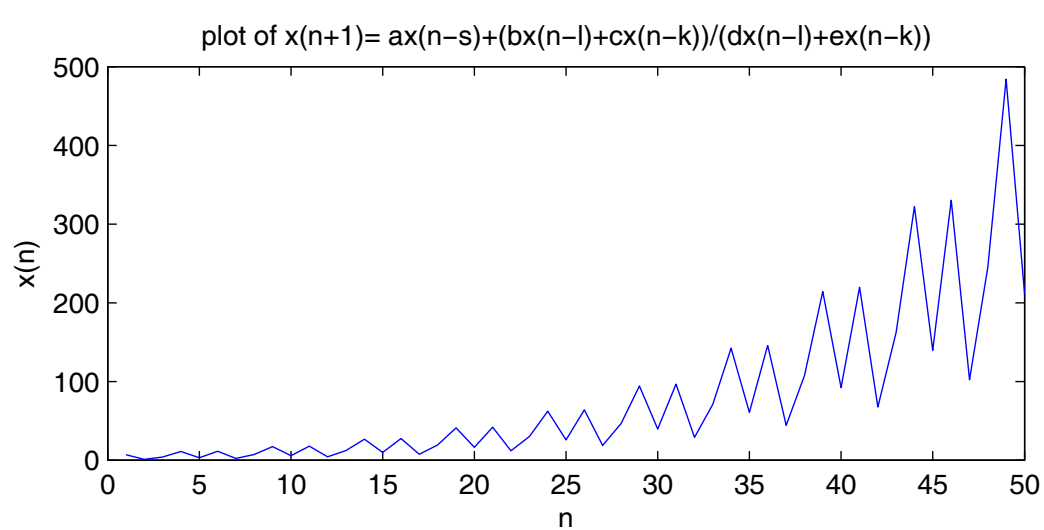

Figure 3 This figure shows the behavior of the solution of Equation (1) when we take $I=2, k=3$, $s=4, x_{-3}=7, x_{-3}=1, x_{-2}=4, x_{-1}=11, x_{0}=3, a=1.5, b=2, c=3, d=3, e=2$.

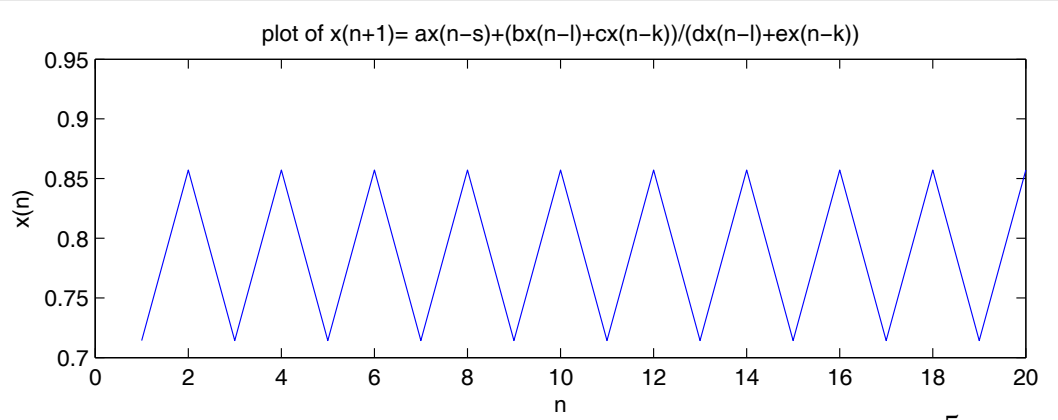
Figure 4 This figure shows the periodicity of the solution of $x_{n+1}=-x_{n-2}+\frac{5 x_{n-1}+6 x_{n-3}}{3 x_{n-1}+4 x_{n-3}}$
since $x_{-3}=x_{-1}=b /(d+e), x_{-2}=x_{0}=c /(d+e)$. 


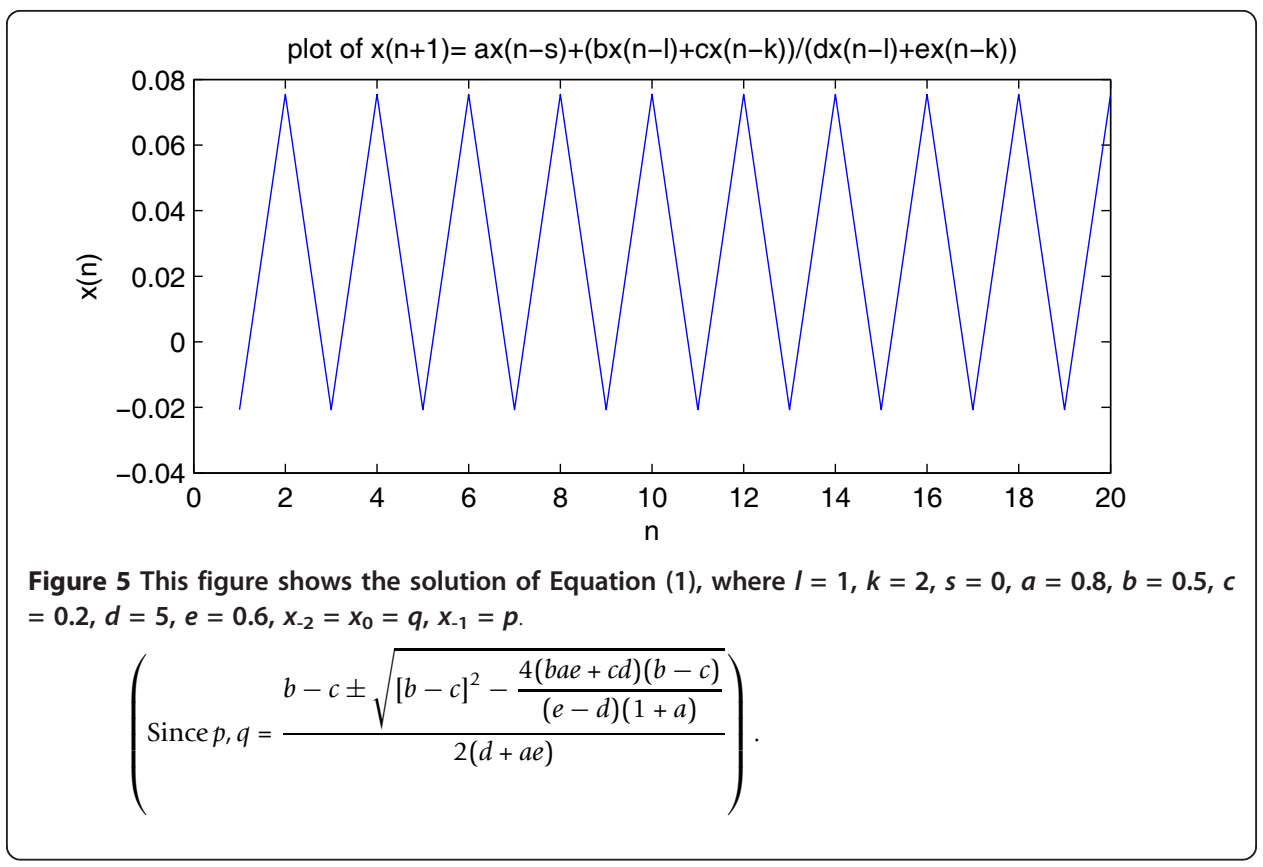

\section{Acknowledgements}

This article was funded by the Deanship of Scientific Research (DSR), the King Abdulaziz University, Jeddah. The authors, therefore, acknowledge with thanks DSR technical and financial support.

\section{Author details}

${ }^{1}$ Mathematics Department, Faculty of Science, King Abdulaziz University, P. O. Box 80203, Jeddah 21589, Saudi Arabia ${ }^{2}$ Department of Mathematics, Faculty of Science, Mansoura University, Mansoura 35516, Egypt

\section{Authors' contributions}

The authors declare that the study was realized in collaboration with the same responsibility. All authors read and approved the final manuscript.

\section{Competing interests}

The authors declare that they have no competing interests.

Received: 4 March 2012 Accepted: 28 May 2012 Published: 28 May 2012

\section{References}

1. Agarwal, RP, Elsayed, EM: On the solution of fourth-order rational recursive sequence. Adv Stud Contemp Math. 20(4), 525-545 (2010)

2. Alogeili, M: Dynamics of a rational difference equation. Appl Math Comp. 176(2), 768-774 (2006). doi:10.1016/j. amc.2005.10.024

3. Elabbasy, EM, El-Metwally, H, Elsayed, EM: Global attractivity and periodic character of a fractional difference equation of order three. Yokohama Math J. 53, 89-100 (2007)

4. Elabbasy, EM, El-Metwally, H, Elsayed, EM: On the difference equation $x_{n+1}=a x_{n}-\frac{b x_{n}}{c x_{n}-d x_{n-1}}$. Adv Differ Equ. 2006, 1-10 (2006)

5. Elabbasy, EM, El-Metwally, H, Elsayed, EM: On the difference equations $x_{n+1}=\frac{\alpha x_{n-k}}{\beta+\gamma \Pi_{i=0}^{k} x_{n-i}}$. J Conc Appl Math. 5(2), 101-113 (2007)

6. El-Metwally, H, Grove, EA, Ladas, G, McGrath, : On the difference equation $y_{n+1}=\frac{y_{n-(2 k+1)}+p}{y_{n-(2 k+1)+q y_{n-2 l}}}$. Proceedings of

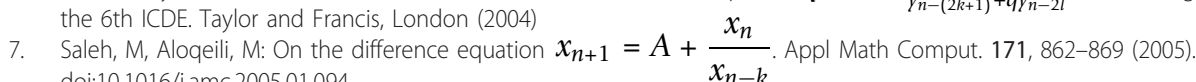
doi:10.1016/j.amc.2005.01.094

8. Simsek, D, Cinar, C, Yalcinkaya, I: On the recursive sequence $x_{n+1}=\frac{x_{n-3}}{1+x_{n-1}}$. Int J Contemp Math Sci. 1(10), 475-480 (2006)

9. Yalçınkaya, I: On the difference equation $x_{n+1}=\alpha+\frac{x_{n-m}}{x_{n}^{k}}$. Discr Dyn Nat Soc. 2008, 8 (2008)

10. Yalçınkaya, I, Cinar, C: On the dynamics of the difference equation $x_{n+1}=\frac{a x_{n-k}}{b+c x_{n}^{p}}$. Fasciculi Mathematici. 42, 133-139

11. Zayed, EME, El-Moneam, MA: On the rational recursive sequence $x_{n+1}=\frac{\alpha+\beta x_{n}+\gamma x_{n-1}}{A+B x_{n}+C x_{n-1}}$. Commun Appl Nonlinear Anal. 12(4), 15-28 (2005)

12. Zayed, EME, El-Moneam, MA: On the rational recursive sequence $x_{n+1}=a x_{n}-\frac{b x_{n}}{c x_{n}-d x_{n-k}}$. Commun Appl Nonlinear Anal. 15, 47-57 (2008) 
13. Agarwal, RP: Difference Equations and Inequalities, 1st edn. Marcel Dekker, New York (1992), 2 (2000)

14. Cinar, C: On the positive solutions of the difference equation $x_{n+1}=\frac{a x_{n-1}}{1+b x_{n} x_{n-1}}$. Appl Math Comp. 156, 587-590 (2004). doi:10.1016/j.amc.2003.08.010

15. Cinar, C: On the positive solutions of the difference equation $x_{n+1}=\frac{x_{n-1}}{-1+a x_{n} x_{n-1}}$. Appl Math Comp. 158(3), 793-797 (2004). doi:10.1016/j.amc.2003.08.139

16. Elabbasy, EM, El-Metwally, H, Elsayed, EM: On the periodic nature of some max-type difference equations. Int J Math Math Sci. 2005(14), 2227-2239 (2005). doi:10.1155/IJMMS.2005.2227

17. Elabbasy, EM, El-Metwally, H, Elsayed, EM: Global behavior of the solutions of difference equation. Adv Diff Equ. 2011, 28 (2011). doi:10.1186/1687-1847-2011-28

18. Elabbasy, EM, El-Metwally, H, Elsayed, EM: Some properties and expressions of solutions for a class of nonlinear difference equation. Utilitas Math. 87, 93-110 (2012)

19. Elabbasy, EM, Elsayed, EM: Dynamics of a rational difference equation. Chinese Ann Math Ser B. 30(2), 187-198 (2009). doi:10.1007/s11401-007-0456-9

20. Elabbasy, EM, Elsayed, EM: Global attractivity and periodic nature of a difference equation. World Appl Sci J. 12(1), 39-47 (2011)

21. El-Metwally, H: Global behavior of an economic model. Chaos Solitons Fract. 33, 994-1005 (2007). doi:10.1016/j. chaos.2006.01.060

22. El-Metwally, H, El-Afifi, MM: On the behavior of some extension forms of some population models. Chaos Solitons Fract. 36, 104-114 (2008). doi:10.1016/j.chaos.2006.06.043

23. Elsayed, EM: A solution form of a class of rational difference equations. Int J Nonlinear Sci. 8(4), 402-411 (2009)

24. Elsayed, EM: Behavior of a rational recursive sequences. Studia Univ "Babes-Bolyai" Mathematica. LVI(1), 27-42 (2011)

25. Elsayed, EM: On the difference equation $x_{n+1}=\frac{x_{n-5}}{-1+x_{n-2} x_{n-5}}$. Int J Contemp Math Sci. 3(33), 1657-1664 (2008)

26. Elsayed, EM: On the Global attractivity and the solution of recursive sequence. Studia Scientiarum Mathematicarum Hungarica. 47(3), 401-418 (2010). doi:10.1556/SScMath.2009.1139

27. Elsayed, EM: Solution and attractivity for a rational recursive sequence. Discr Dyn Nat Soc. 2011, 17 (Article ID 982309) (2011)

28. Elsayed, EM: Qualitative behavior of difference equation of order three. Acta Scientiarum Mathematicarum (Szeged). 75(1-2), 113-129 (2009)

29. Elsayed, EM: Qualitative behavior of difference equation of order two. Math Comput Model. 50, 1130-1141 (2009). doi:10.1016/j.mcm.2009.06.003

30. Elsayed, EM: Solutions of rational difference system of order two. Math Comput Model. 55, 378-384 (2012). doi:10.1016/ j.mcm.2011.08.012

31. Elsayed, EM: On the solution of some difference equations. Eur J Pure Appl Math. 4(3), 287-303 (2011)

32. Elsayed, EM: On the dynamics of a higher order rational recursive sequence. Commun Math Anal. 12(1), 117-133 (2012)

33. Elsayed, EM, El-Dessoky, MM, Alotaibi, A: On the solutions of a general system of difference equations. Discr Dyn Nat Soc 2012, 12 (2012). (Article ID 892571)

34. Kocic, VL, Ladas, G: Global Behavior of Nonlinear Difference Equations of Higher Order with Applications. Kluwer Academic Publishers Dordrecht (1993)

35. Kulenovic, MRS, Ladas, G: Dynamics of Second Order Rational Difference Equations with Open Problems and Conjectures. Chapman \& Hall/CRC Press, Boca Raton (2001)

36. Rafiq, A: Convergence of an iterative scheme due to Agarwal et al. Rostock Math Kolloq. 61, 95-105 (2006)

37. Saleh, M, Abu-Baha, S: Dynamics of a higher order rational difference equation. Appl Math Comput. 181, 84-102 (2006). doi:10.1016/j.amc.2006.01.012 38. Saleh, M, Aloqeili, M: On the difference equation $y_{n+1}=A+\frac{y_{n}}{y_{n-k}}$ with $A<0$. Appl Math Comput. 176(1), 359-363
(2006). doi:10.1016/j.amc.2005.09.023

39. Touafek, N, Elsayed, EM: On the solutions of systems of rational difference equations. Math Comput Model. 55, 1987-1997 (2012). doi:10.1016/j.mcm.2011.11.058

40. Wang, C, Wang, S, Li, L, Shi, Q: Asymptotic behavior of equilibrium point for a class of nonlinear difference equation. Adv Diff Equ. 2009, 8 (2009)

41. Wang, C, Shi, Q, Wang, S: Asymptotic behavior of equilibrium point for a family of rational difference equation. Adv Diff Equ. 2010, 10 (2010)

42. Wang, C, Wang, S, Wang, Z, Gong, H, Wang, R: Asymptotic stability for a class of nonlinear difference equation. Discr Dyn Nat Soc. 2010, 10 (2010)

43. Wang, C, Wang, S, Wang, W: Global asymptotic stability of equilibrium point for a family of rational difference equations. Appl Math Lett. 24(5), 714-718 (2011). doi:10.1016/j.aml.2010.12.013

44. Yalçınkaya, I: On the global asymptotic stability of a second-order system of difference equations. Discr Dyn Nat Soc. 2008, 12 (2008)

45. Yalçınkaya, I, Cinar, C, Atalay, M: On the solutions of systems of difference equations. Adv Diff Equ. 2008, 9 (2008)

doi:10.1186/1687-1847-2012-69

Cite this article as: Elsayed and El-Dessoky: Dynamics and behavior of a higher order rational recursive sequence.

Advances in Difference Equations 2012 2012:69. 